\title{
CORRECTION
}

\section{Correction to: Topological localization approach for efficient energy management of WSN}

\author{
Hitesh Mohapatra ${ }^{1,2}$ (1) $\cdot$ Amiya Kumar Rath ${ }^{2,3}$ (1) $\cdot$ Rakesh K Lenka $^{4} \cdot$ Rudra Kalyan Nayak $^{1} \cdot$ Ramamani Tripathy $^{5}$
}

(c) Springer-Verlag GmbH Germany, part of Springer Nature 2021

Correction to: Evolutionary Intelligence

https://doi.org/10.1007/s12065-021-00611-z

Unfortunately, one of the author (Rudra Kalyan Nayak) affiliation was published incorrectly. The correct affiliation is given below.

The original article has been corrected.

Rudra Kalyan Nayak ${ }^{1}$

${ }^{1}$ Department of Computer Science and Engineering, Koneru Lakshmaiah Education Foundation, Vaddeswaram, Guntur, AP, 522502, India

Publisher's Note Springer Nature remains neutral with regard to jurisdictional claims in published maps and institutional affiliations.

The original article can be found online at https://doi.org/10.1007/ s12065-021-00611-z.

Hitesh Mohapatra

hiteshmahapatra@gmail.com

1 Department of Computer Science and Engineering, Koneru Lakshmaiah Education Foundation, Vaddeswaram, Guntur, AP 522502, India

2 Department of Computer Science \& Engineering, Veer Surendra Surendra Sai University of Technology, Burla, OD, India

3 NAAC, Banglore, India

4 Department of Computer Science \& Engineering, IIIT, Bhubaneswar, OD, India

5 Dept.of Master of Computer Application, United School of Business Management, Bhubaneswar, OD, India 